\begin{tabular}{|c|c|}
\hline \multirow{3}{*}{ 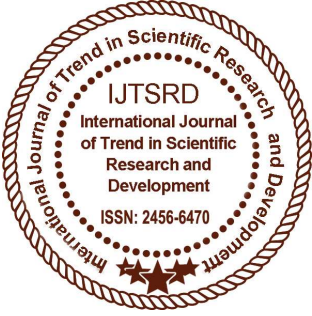 } & $\begin{array}{l}\text { International Journal of Trend in Scientific } \\
\text { Research and Development (IJTSRD) }\end{array}$ \\
\hline & International Open Access Journal \\
\hline & ISSN No: 2456 - 6470 | www.ijtsrd.com | Volume - 2 | Issue - 4 \\
\hline
\end{tabular}

\title{
An Augmented Implementation of Ad hoc On-Demand Distance Vector (AODV) Routing for Internet of Things (IoT)
}

\author{
Mohd Muntjir, Mohd Rahul \\ Department of Information Technology College of Computers and Information Technology, \\ Taif University, Taif, Kingdom of Saudi Arabia
}

\section{ABSTRACT}

IoT has grown from the convergence of wireless technologies, micro-electro mechanical systems, micro-services and the Internet. The convergence has improved tear down the silo walls between operational technology and information technology, consenting amorphous machine-generated data to be evaluated for insights that will enterprise developments. Designing of routing protocols for the Internet of things is an important part in the research of Internet of Things. The Internet of things is a new concept in IT field, but the research of routing protocols for Internet of Things is still blank. We have improved the AODV (Ad hoc On-Demand Distance Vector) algorithm through optimizing the protocol, such as we combined together routing table and the Internet connecting table. In this project, we have designed a routing method that can take as routing destination not just like nodes. This type of improvement is more suitable for the use in the Internet of things, by connecting to the Internet. At the end of this paper, simulation results showed that AODV-IOT achieves better results in packet delivery ratio, average end-to-end delay, throughput and consumed energy.

Keywords: IoT, Thing's Information as Object, Utilities, Smart business, 3-layer architecture of IoT, $A O D V$

\section{INTRODUCTION}

\section{A. Internet of Things (IoT):}

The Internet of Things is a term devised by Kevin Ashton, working on RFID who perceived an organization of pervasive sensors connecting the physical world to the Internet. Though things, Internet, and connectivity are the three main factors of The Internet of Things (IoT). The Internet of Things (IoT) is transformational force that can aid businesses expand enactment through Internet of Things analytics and IoT Security to bring improved consequences [11].

Internet of Things (IOT) involves to the networked interconnection of everyday objects. It is generally viewed/as a self-configuring wireless network of sensors whose function would be to interconnect all things [1].

It qualifies devices to perceive, classify and comprehend a situation or the environments without being supported on individual advantage. There are physical objects one wants to be able to track, to monitor and to relate with. Examples include inanimate objects like pallets, boxes containing consumer goods, cars, machines, fridges -and perhaps even the infamous carton of milk or cup of yoghurtas well as animate things like animals and humans. These are the things of the Internet of Things - or to use a clearer term, the entities of interest [2]. Many experts are optimistic about the future of Internet of Things, while several of them contemplate on the habit of IOT. Nonetheless, as crucial parts of IOT the routing method designing is still a blank [1]. In figure 
1 a thing of interest is monitored by advice in the environment, or it can also have a device attached [2].

\section{Basic Attribute of IOT:}

Language is a reflection of the existence, and word is significant to the entity. The real meaning of phrase Internet of things corresponds to the basic attributes of entity Internet of Things. The semantic meaning "Internet of thing's information" points out the two basic attributes of the entity that it denotes: the one is "being an Internet", the other is "linking to thing's in sequence" [7].

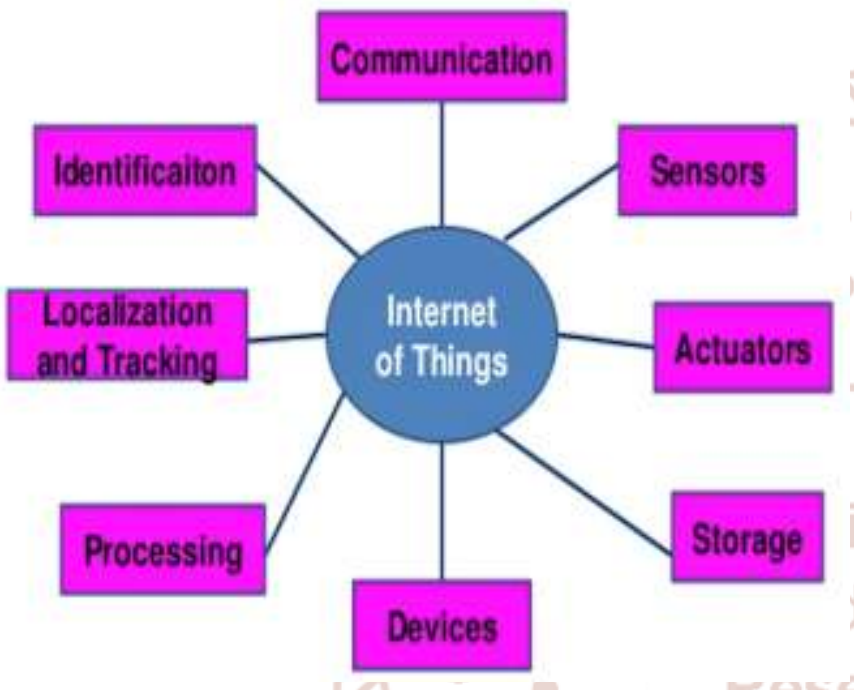

Fig.2. Components of IoT

\section{A Novel kind of Internet Application:}

Every entity is composed of a structure layer and a function layer. The structure is a base sustaining the function, and the function is implemented on the structure. According to a different context, an entity's name designates its structure, or its function, or both of them. The structure of Internet is a physical system composed of many computers that linked each other by some transmission medium; the function of Internet is to broadcast information supplied by some agents for hoping to communicate with others [12].

\section{Thing's Information as an Object:}

Things including substances and products are the bases of human living, those main characters are two: large quantity and various types; relative fixed material entity. The former demands an effective management to the thing's information, the lastly defines that the object's information can be shown in relative fixed form, so it can be recorded on some material medium, and adhered to the thing's entity. The things information and the thing's entity are combined into one [5].

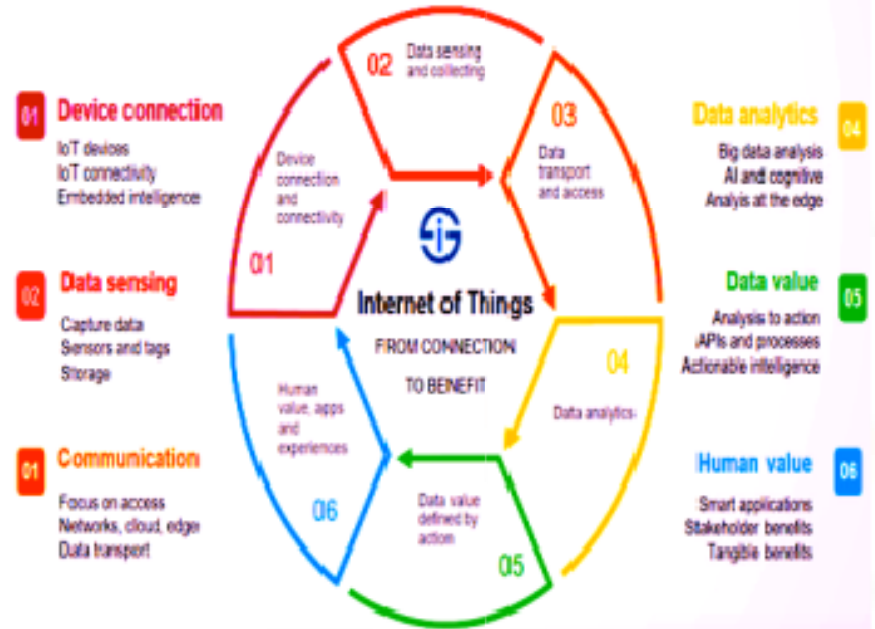

Fig.3. The Internet of Things: From Connecting Devices to Human Value

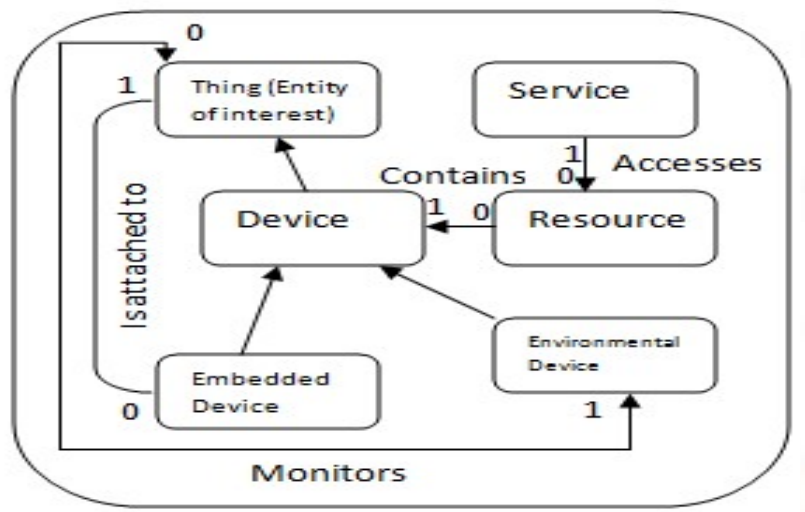

Fig.4. Relationship among things, devices, resources and services

\section{Application of IoT:}

According to Fleisch, The IoT (Internet of Things) is relevant in every step in every value chain. He has notorious seven major value drivers. The first four based on value from machine-to-machine communication, while the last three create value with the mixing of users. The drivers as identified by Fleisch are:

Simplified manual proximity trigger- things can correspond their identity when they are placed into the sensing space of a sensor. Once the identity is recognized and communicated, a definite action or transaction can be triggered.

Automatic proximity trigger- an action is triggered repeatedly when the physical distance of two things drops under (or passes) a threshold. The identity of the thing is identified, which when joint with the physical location and action allows for good processes. 
Automatic sensor triggering- a smart (or cooperative) thing can gather data via any type of sensor consisting temperature, acceleration, orientation, vibration and humidity. The thing observes its situation and environment, communicates the information, which allows prompt (and global) decision-making.

Automatic product security- a thing can supply derived security information based on the interaction between the thing and its cyberspace demonstration (e.g. a QR-code containing a specific URL pointing to relevant information)[3]. There are several application domains, which will be compressed by the emergence of Internet of things. The application can be classified into the diverse network availability, scale, coverage and repeatability of use involvement. mechanism to abridge routine work life and personal tasks [11].

\section{3-layer architecture of loT:}

Poles apart from its definition unclear, the architecture of loT are generally accepted. The well-known 3-layer architecture contains of the Perception Layer, the Network Layer and the Application layer, as shown in given Figure.

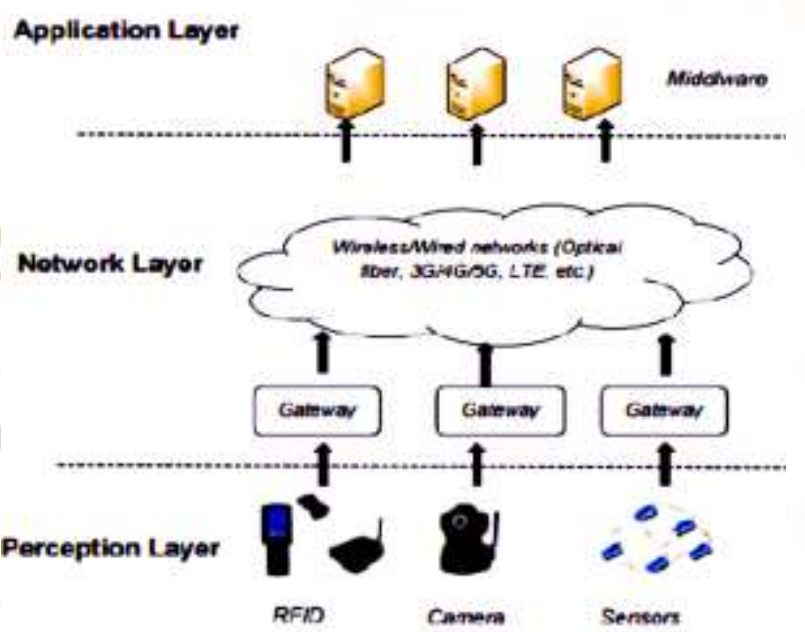

Fig.6. 3-layer architecture of the Internet of Things [4]

\section{The Perception Layer:}

In the perception layer, the Perception Layer system aims to acquire, collect and process the data from the physical world [10]. The Perception Layer is similar to the facial skin and the five sense organs of IOT, which helps mainly in identifying objects and gathering information. The Perception Layer consists 2-D bar code labels and readers, RFID tags and reader-writers, terminals, camera, GPS, sensors, and sensor network. Its main task is to identify the object, gathering information [4].

\section{The network layer:}

In the Network layer, the system aims to transfer data in a large area or long distance. The network layer is like the neural network and brain of IOT, its main function is to transmit and process the information. The network layer includes a convergence network of communication and Internet network, in sequence center, network management center, and intelligent processing center, etc. The network layer will transmit and process the information produced from perception layer.

\section{The Application Layer:}

Data processing and Application Layer services providing are two major purposes of the application layer [10]. The Application Layer is a combination of 
1OT's social division and industry demand, to realize the extensive intellectualization. The Application Layer is the deep convergence of 1OT and business technology, combined with industry needs to realize the intellectualized industry, which is related to person's social division of labor, eventually form human society [6].

\section{"Consumer" loT 2-Layered Architecture}
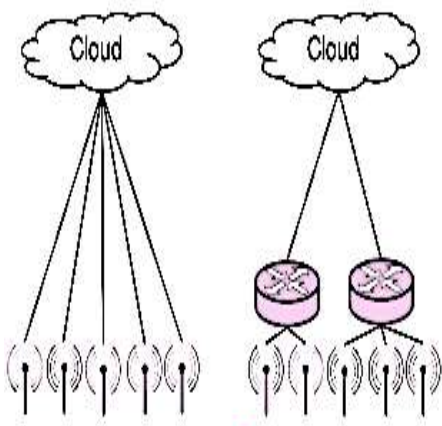

$=$

Fig.7. Consumer vs Industrial IoT
Industrial loT
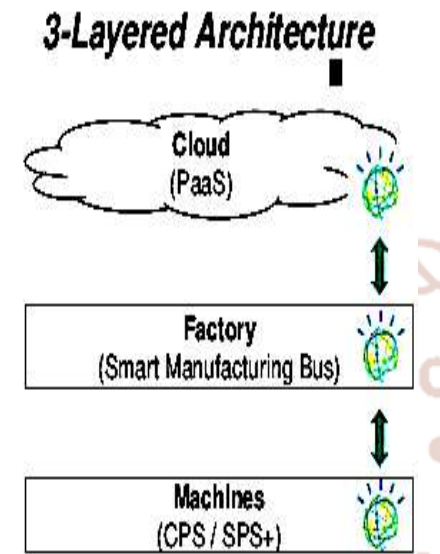

(CPS/SPS+)

\section{Related Work}

Ad hoc On-Demand Distance Vector Routing (AODV) is a responsive hop-by-hop routing protocol. Two beneficiaries of AODV have been established since its speciation: The Lightweight On demand Ad hoc Distance-vector Routing Protocol - Next Generation (LOADng) and AODVv2, with the latter having been approved by the MANET working group of the Internet Engineering Task Force. Though AODV only admits Hop Count as a metric, both of its successors permit alternate metrics, opening up the opportunity for arrangement of an energy-aware metric.

The research work performed in this area by different researchers is presented as follows:

Yicong Tianet al. Internet of Things is a rather new concept in IT field. The However, the research of routing protocols of Internet of Things is still a empty, while route designing is an important part in the research of Internet of Things. In this method, author designed a routing method that can take function as routing destination not just nodes. The development is more suitable for the use in Internet of things, which most unique character is connecting to the internet. Interrelate with AOMDV in the Internet of things, simulation results show that AOMDV-IOT achieves improved performance in average end-to-end delay, packet loss and discovery frequency.

Stephan Haller et al. The Internet of Things is a hyped word and many definitions for it exist. Worse still, it comes with a lot of related terminology that is not used regularly either, hindering scientific discourse. This paper tries to bring clarity by describing the most significant terms like things, devices, and entities of interest, resources, addressing, and identity and, more importantly, the relationships between them.

Yinghui Huang et al. From a semantic analysis for Internet of things, a conclusion is drawn that the word thing here indicates essentially the thing's information and the Internet here is actually the Internet application. Internet of Things is a new kind of Internet application, which makes the thing's information be common on a global scale. Internet of Things has two attributes: being an Internet application and dealing with thing's information, and four discrepancy features: only for thing's information, coded by UID or EPC, stored in RFID electronic tag, uploaded by non-contact analysis with RFID reader. The descriptive models for Internet of Things are introduced based on the vital attributes and the differential features. The graphical model of Internet of Things shows that the two key tasks of constructing Internet of Things are the preprocessing of thing's information before uploaded into Internet and the building of a RFID system. The rest expression model of Internet of Things stresses the common character all Internet applications have (explicitly an Internet application is essentially a set of information), so the improvement processes and the technical methods of other Internet applications could be referenced and reused for building Internet of Things.

Miao Wu et al. The Internet of Things is a technological revolution that represents the future of computing and connections. It is not the simple extension of the Internet or the Telecommunications Network. .It has the features of two, the Internet and the Telecommunications Network, and also has its own distinguishing feature. Through analyzing the present accepted three-layer structure of the Internet of things, author suggests that the three-layer structure can't express the entire features and connotation of the Internet of Things. After reanalyzing the technical structure of the Internet and the Logical Layered 
Architecture of the Telecommunication Management Network, author establishes new five-layer construction of the Internet of Things. The author believes this architecture is more easy and helpful to comprehend the essence of the Internet of Things, and hoping it is helpful to develop the Internet of Things.

Lu Tan et al. Nowadays, the main communication form on the Internet is human-human. But it is foreseeable that in a close soon that any object will have a unique way of identification and can be addressed so that every object can be linked. The Internet will become to the Internet of Things. The communicate forms will develop from human-human to human-human, human-thing and thing-thing (also calledM2M). This will bring a new ubiquitous computing and communication age and change people's life extremely. Radio Frequency Identification techniques (RFID) and related recognition technologies will be the cornerstones of the upcoming Internet of Things (IOT). This paper target to show a skeleton of the Internet of Things and we try to address some essential issues of the Internet of Things like its construction and the interoperability, etc. At the beginning we describe an overview of the Internet of Things. Then author give our architecture design proposal of the Internet of Things and then we design a specific the Internet of Things application model that can relate to automatic facilities management in the smart campus. At last, talk about some open questions about the Internet of Things

Zhiyong Shi et al. The Internet of things is a new generation information network, which realizes machine-to-machine communication. In view of the fact that people must the mobility of the Internet of things, the integration scheme of TD-SCDMA network and the Internet of things is intended.3G TDSCDMA network is used as the basic network of transmitting information of the Internet of things. At the same time, TD-SCDMA mobile terminal is integrated with RFID reader. TD-SCDMA networks can supply high-bandwidth and high-speed information transmission channel for the Internet of things. TD-SCDMA mobile terminal can recognize the mobility of the access part for the Internet of things. Additionally, the network element functions of the mobile Internet of effects are described. In order to realize the information exchange involving TDSCDMA network and the Internet of things, the communication protocols of the mobile Internet of things are discussed and designed in detail. Lastly, the network performance, which is gotten by using OPNET software, shows the mobile Internet of things placed on TDSCDMA network can be realized by adopting the rational network design and the effective communication protocols.

Louis Coetzee et al. Internet is a living entity, always altering and evolving. New applications and businesses are formed continuously. In addition to an evolving Internet, technology is also altering the landscape. Broadband connectivity is becoming cheap and ubiquitous; devices are becoming more powerful and smaller with a range of on-board sensors. The proliferation of more devices becoming connected is foremost to a new standard: the Internet of Things. The Internet of Things is driven by an expansion of the Internet through the inclusion of physical objects united with an ability to provide smarter services to the environment as more data becomes available. Diverse application domains ranging from Green-IT and energy efficiency to logistics are already starting to profit from Internet of Things concepts. There are challenges associated with the Internet of Things, most explicitly in areas of trust and safety, standardization and governance required to ensure a fair and trustworthy open Internet of Things which supplies value to all of society. Internet of Things is high on the research agenda of several multinationals and also the European Commission and countries like as China. The research conducted is driving the creation of a useful and powerful Internet of Things. The payback of Internet of Things to the developing and emerging economies are momentous, and strategies to realize these need to be found.

Anamika Sharma et al. has proposed a protocol based on AODV to improve the routing of AODV for Internet of things. It is modified AODV to adapt with the usage in Internet of things. Our principal objective is to find and create the connection between nodes and Internet efficiently. The routing protocol will find the most appropriate link automatically, and record other links as back up. If a node need to create a link to the internet, it ought to first check its internet connecting table, if the information there is valid, the node will choose the node which hops count is the lowest, or start the routing finding process. Then it will take the discovered node as destination node to send message. In this paper AODV routing protocol is improved for the Internet of things. In order to improve the algorithm the protocol is optimized such that routing table and Internet connecting table will 
combine into one [9]. If a node need to generate a link to the internet, it should initial confirm its internet connecting table, if the information there is effective, the node will choose the node whose hops count is lowest or start the route finding process. Then it will take the determined node as purpose node to send message.

Peng-fei FAN et al. in this paper author analyzes the application of the internet of thing technology in postal logistics, forecast the potential structure of the technology of Internet of Things in postal logistics and its impact on the production of the business model of Internet of Things. It primarily analyses the core way for the technology of Internet of Things to make productivity in postal logistics and discusses the current business models in the point of management and decision making of the industry of Internet of Things. The paper also studies the feature of the business models of Internet of Things in postal logistics combining features of latest industry of Internet of Things and characteristics of the postal logistics, puts onward a viable business model, and makes a organized exposition of the advantage of this model [8].

\section{Proposed Work \\ A. Problem Formulation}

For improving the routing of AODV for Internet of Things we are working on the implementation of the algorithm through optimizing the protocol, such as routing table and Internet connecting table will combine into one. Additionally, the improvement protocol not only can use in Internet of things, but also some other fields. Now we can use our advanced protocol to search the function, and the algorithm will find appropriate node for us.

\section{Proposed Work}

In this dissertation we have proposed a protocol based on AODV to improve the routing of AODV for Internet of things. It is modified AODV to adapt with the usage in Internet of things. Our principal objective is to find and create the connection between nodes and Internet efficiently.

\section{Results And Analysis}

Here we compare the results of AODV and AODVIOT. The comparison is done for 10,20 and 30 nodes. The results of AODV and AOMDV-IOT for 10 nodes are shown in given tables.
Table 1: Result of default AODV for node (n) $=10$

\begin{tabular}{|l|l|}
\hline $\begin{array}{l}\text { Result of default AODV } \\
\text { for node (n) = 10 }\end{array}$ \\
\hline Total Packets Sent & 9994 \\
\hline Total Packets Received & 999 \\
\hline Total Packets Dropped & 10 \\
\hline Packet Delivery Ratio & $99.98 \%$ \\
\hline $\begin{array}{l}\text { Throughput of the } \\
\text { Network (kbps) }\end{array}$ & 4.9961 \\
\hline Average End to End Delay & $0.082337373 \mathrm{~ms}$ \\
\hline Total Energy Consumption & 17.6629 \\
\hline
\end{tabular}

Table 2: Result of default AODV for node (n) $=10$

\begin{tabular}{|l|l|}
\hline $\begin{array}{l}\text { Result of default AODV- } \\
\text { IOT for node (n) }=\mathbf{1 0}\end{array}$ \\
\hline Total Packets Sent & 9994 \\
\hline Total Packets Received & 9994 \\
\hline Total Packets Dropped & 0 \\
\hline Packet Delivery Ratio & $100.00 \%$ \\
\hline $\begin{array}{l}\text { Throughput of the } \\
\text { Network (kbps) }\end{array}$ & 4.9970 \\
\hline Average End to End Delay & $0.001381516 \mathrm{~ms}$ \\
\hline Total Energy Consumption & 13.7340 \\
\hline
\end{tabular}

The comparison of performance metrics for AODV and AOMV-IOT for 10 nodes is shown in given figure.

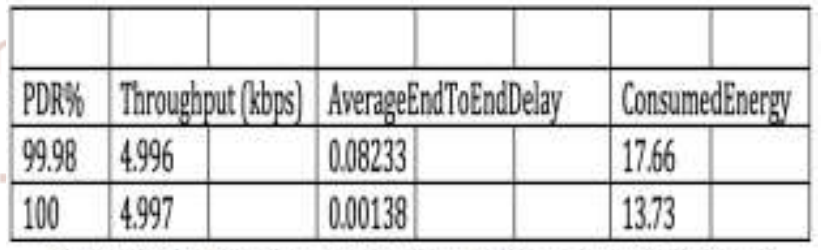

The Result of AODV and AOMDV.1OT for 10 nodes are shown in This Figure

Fig.8. Performance Metrics for nodes $(n)=10$

The results of AODV and AOMDV-IOT for 20 nodes are shown in given tables.

Table 3: Result of default AODV for node $(n)=20$

\begin{tabular}{|l|l|}
\hline $\begin{array}{l}\text { Result of default AODV } \\
\text { for node (n) }=\mathbf{2 0}\end{array}$ \\
\hline Total Packets Sent & 6900 \\
\hline Total Packets Received & 6895 \\
\hline Total Packets Dropped & 47 \\
\hline Packet Delivery Ratio & $99.91 \%$ \\
\hline $\begin{array}{l}\text { Throughput of the } \\
\text { Network (kbps) }\end{array}$ & 3.3986 \\
\hline Average End to End Delay & $0.004611620 \mathrm{~ms}$ \\
\hline Total Energy Consumption & 30.6230 \\
\hline
\end{tabular}

Table 4: Result of AODV-IOT for node $(\mathrm{n})=20$ 
International Journal of Trend in Scientific Research and Development (IJTSRD) ISSN: 2456-6470

\begin{tabular}{|l|l|}
\hline $\begin{array}{l}\text { Result of default AODV- } \\
\text { IOT for node (n)= 20 }\end{array}$ \\
\hline Total Packets Sent & 6900 \\
\hline Total Packets Received & 6798 \\
\hline Total Packets Dropped & 2 \\
\hline Packet Delivery Ratio & $99.98 \%$ \\
\hline $\begin{array}{l}\text { Throughput of the } \\
\text { Network (kbps) }\end{array}$ & 3.4097 \\
\hline Average End to End Delay & $\begin{array}{l}0.001523509 \\
\mathrm{~ms}\end{array}$ \\
\hline Total Energy Consumption & 21.6010 \\
\hline
\end{tabular}

The comparison of performance metrics for AODV and AOMV- IOT for 20 nodes is shown in given figure.

\begin{tabular}{|l|l|l|l|l|l|l|l|}
\hline & & & & & & \\
\hline PDR\% & \multicolumn{2}{|l|}{ Throughput(kops) } & \multicolumn{2}{|l|}{ AverageEndToEndDelay } & \multicolumn{2}{|l|}{ ConsumedEnergy } \\
\hline 99.91 & 3.398 & & 0.00461 & & & 30.62 & \\
\hline 99.98 & 3.409 & & 0.00152 & & & 21.60 & \\
\hline
\end{tabular}

The Result of AODV and AOMDV-10T for 20 nodes are shown in This Figure

Fig.9. Performance Metrics for nodes $(n)=20$

The results of AODV and AOMDV-IOT for 30 nodes are shown in given tables.

Table 5: Result of default AODV for node $(n)=30$

\begin{tabular}{|l|l|}
\hline $\begin{array}{l}\text { Result of default AODV } \\
\text { for node (n)= 30 }\end{array}$ & 5430 \\
\hline Total Packets Sent & 5370 \\
\hline Total Packets Received & 90 \\
\hline Total Packets Dropped & $99.21 \%$ \\
\hline Packet Delivery Ratio & 2.6915 \\
\hline $\begin{array}{l}\text { Throughput of the } \\
\text { Network (kbps) }\end{array}$ & \\
\hline Average End to End Delay & $0.055677854 \mathrm{~ms}$ \\
\hline Total Energy Consumption & 46.3231 \\
\hline
\end{tabular}

Table 6: Result of AODV-IOT for node $(n)=30$

\begin{tabular}{|l|l|}
\hline $\begin{array}{l}\text { Result of default AODV- } \\
\text { IOT for node (n) }=\mathbf{3 0}\end{array}$ \\
\hline Total Packets Sent & 5430 \\
\hline Total Packets Received & 5413 \\
\hline Total Packets Dropped & 1 \\
\hline Packet Delivery Ratio & $99.99 \%$ \\
\hline $\begin{array}{l}\text { Throughput of the } \\
\text { Network (kbps) }\end{array}$ & 2.7187 \\
\hline Average End to End Delay & $0.001735674 \mathrm{~ms}$ \\
\hline Total Energy Consumption & 31.8790 \\
\hline
\end{tabular}

The comparison of performance metrics for AODV and AOMV-IOT for 30 nodes is shown in given figure.

\begin{tabular}{|c|c|c|c|c|c|c|}
\hline PDRY & \multicolumn{2}{|c|}{ Throughput(kbps) } & \multicolumn{2}{|c|}{ AverageEndT'TEndDelay } & \multicolumn{2}{|c|}{ Consumedinerg } \\
\hline 99.21 & 2.691 & & 0.0556 & & 46.32 & \\
\hline 99.99 & 2718 & & 0.0017 & & 31.87 & \\
\hline
\end{tabular}

Fig.10. Performance Metrics for nodes (n)=30

\section{Conclusion And Future Scope}

Internet of Things has the widespread future with providing applications with many aids to users. The standard reactive routing protocols such as AODV and is designed to find just the shortest path without any concern of the energy consumption of a node. Internet of Things has prominent potential of benefits over the worldwide. As every new technology has some challenges, Internet of Things has also some challenges .The present has discussed AODV routing protocol for Internet of Things. Here we improved the algorithm through optimizing the protocol, such as routing table and Internet connecting table are combined into one. Application concerned study is needed for different industrial application in which IOT can be used in order to start a new technological revolution. New security experiments and use of lightweight cryptographic protocol need to be considered further. The results, uncover that the proposed protocol is efficiently able to counter the nodes in three distinctive developments.

\section{ACKNOWLEDGEMENT}

We would like to gratefully and sincerely thank to The Dean and Vice Dean of our College and Chairman of our Departments for his guidance, understanding, patience, and most importantly, his friendly nature during this research paper. We would also like to thank my friends and colleagues, and the university who provided me an efficient support to work on this atmosphere and good infrastructure. We would also like to thank to all the previous researchers who worked very hard and helped others to comprehend the subject of AODV and Internet of Things (IoT). 


\section{REFERENCES}

1) Yicong TIAN, Rui HOU, “An Improved AOMDV Routing Protocol for Internet of Things", 2010 IEEE

2) Stephan Haller, "The Things in the Internet of Things", Internet of Things Conference 2010, Tokyo, Japan, http://www.iot2010.org/

3) Yinghui Huang, Guanyu Li, "Descriptive Models for Internet of Things", International Conference on Intelligent Control and Information Processing August 13-15, 2010 - Dalian, China

4) Miao Wu, Ting-lie Lu, Fei-Yang Ling, ling Sun, Hui-Ying Du, "Research on the architecture of Internet of things", 2010 3rd International Conference on Advanced Computer Theory and Engineering (ICACTE)

5) Lu Tan,Neng Wang, "Future Internet: The Internet of Things", 2010-3rd International Conference on Advanced Computer Theory and Engineering (ICACTE).

6) Zhiyong Shi, Kui Liao, Shiping Yin, 'Design and Implementation of the Mobile Internet of Things Based on TD-SCDMA Network", 2010-IEEE

7) Rafael Angarita, "Responsible Objects: Towards Self-healing Internet of Things Applications", 2015-IEEE 12th International Conference on Autonomic Computing.

8) Peng-fei FAN, Guang-zhao ZHOU, "Analysis of the Business Model Innovation of the Technology of Internet of Things in Postal Logistics", 2011IEEE

9) Anamika Sharma, Er. Sonia Saini,"Energy Efficient AODV Protocol for Internet of Things", International Journal of Advanced Research in Electronics and Communication Engineering (IJARECE), ISSN: 2278 - 909X, Volume 5, Issue 8, August 2016,pp-2198-2203

10) http://netlab.csie.ntut.edu.tw/seminar/year2011/99 598001_20111130.pdf

11) https://aws.amazon.com/iot/

12) Mohd Muntjir, Mohd Rahul, Hesham A. Alhumyani,"An Analysis of Internet of Things (IoT): Novel Architectures, Modern Applications, Security Aspects and Future Scope with Latest Case Studies", International Journal of Engineering Research \& Technology (IJERT), ISSN: 2278-0181, Vol. 6 Issue 06, June - 2017, pp: $422-448$
13) Mohd Rahul, Hesham A. Alhumyani, Mohd Muntjir, Minakshi Kamboj, "An Improved Homomorphic Encryption for Secure Cloud Data Storage",(IJACSA) International Journal of Advanced Computer Science and Applications, Vol. 8, No. 12, 2017

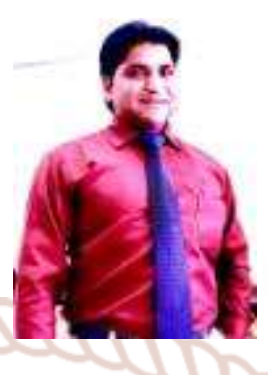

Mohd Muntjir and is a Doctor of Philosophy (Ph.D.) in Computer Science and working in Department of Information Technology, College of Computers and Information Technology Taif University Taif, Kingdom of Saudi Arabia. I am an internationally recognized scholar who has made robust contributions to the comprehension and cognizant of Wireless Sensor Networks, Internet of Things (IoT), Cloud Computing, E-Commerce, Multimedia, E-Business, and Database Management System. I have over ten years of research, teaching, and industry experience at various positions. I have completed many projects, published research papers in reputed international journals/conferences, and edited/co-authored of books and book chapters, and member of international organizations. I am also assisting as an Editorial Board Member in International Journal's editorial board panel and working as a Reviewer of 10 International Journal's reviewer board panel.

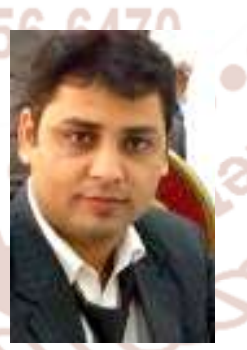

Mohd Rahul is a Doctor of Philosophy (Ph.D.) in Computer Science and working in Department of Information Technology, College of Computers and Information Technology Taif University Taif, Kingdom of Saudi Arabia. Working in Department of Information Technology, College of Computers and Information Technology Taif University at Taif Saudi Arabia. He has obtained his Ph.D. Degree form OPJS University Rajasthan India. Mohd Rahul received M.C.A. degree from Punjab Technical University Jalandhar, India and M.Tech (IT) degree from KSO University Karnataka, India. His research interests are Cloud Computing, Computer Networks, routing protocols, and IoT (Internet of Things). Mohd Rahul has published many research papers in distinctive journals, conferences and books and book Chapters. 